\title{
El nuevo modelo de gestión educativa y su impacto en las escuelas de educación básica del cantón Cañar, Ecuador
}

\section{The new model on educational management and its impact on the education in primary schools in the parish of Cañar, Ecuador}

\author{
Marcos Eduardo Cantos Ochoa ${ }^{1 *}$ y Johanna Rosalí Reyes Reinoso ${ }^{1}$ \\ ${ }^{1}$ Universidad Católica de Cuenca, Sede Cañar \\ *mecantoso@ucacue.edu.ec
}

DOI: https://doi.org/10.26871/killkana_social.v2i4.100

\begin{abstract}
Resumen
El presente estudio tiene por objetivo evaluar el nuevo modelo de gestión educativa implementado en el Ecuador y su impacto en las escuelas de educación básica del cantón Cañar - Ecuador. Este modelo constituye un paradigma de organización interna y externa a nivel institucional que permite canalizar en forma efectiva y oportuna, las directrices a ser aplicadas dentro del campo educativo desde diferentes ámbitos; se plantea una nueva reestructuración que garantice y asegure el cumplimiento del derecho a la educación, un camino que conducirá a la calidad y calidez para fortalecer los servicios educativos atendiendo las realidades locales y culturales, impactando de sobremanera en las escuelas de educación básica. La investigación está dividida en dos tipos: descriptiva, ya que se aplicó un cuestionario de 10 preguntas a 124 docentes pertenecientes a las escuelas: Carlos Cueva Tamariz, Luís Roberto Chacón, Simón Bolívar, San José de Calasanz, Ezequiel Cárdenas y Santa Rosa de Lima de la ciudad de Cañar, Ecuador. Documental por el apoyo de la investigación mediante medios electrónicos y bibliográficos. Entre algunas conclusiones que se pueden mencionar tenemos que el nuevo modelo de gestión educativa implementado en el Ecuador en el año 2010, tiene una incidencia importante en las escuelas de educación básica de la ciudad de Cañar, Cantón Cañar - Ecuador, pues sus actores en su gran mayoría conocen sus lineamientos y ratifican el cumplimiento de sus objetivos, especialmente en lo que tiene que ver al fortalecimiento de la calidad de la educación, mediante la actualización y fortalecimiento de los currículos de la Educación General Básica y del Bachillerato y la construcción del currículo de Educación Inicial. Y entre las recomendaciones, compartir responsabilidades y liderazgos para dar cumplimiento al nuevo modelo de gestión educativa y aproximarlos hacia la ciudadanía, atendiendo las realidades locales y culturales para asumir adecuadamente los roles asignados.
\end{abstract}

Palabras clave: Descentralización, Educación, Gestión, Organización.

\begin{abstract}
The purpose of this study was to evaluate the new educational management model implemented in Ecuador and its impact on basic education schools in the Cañar canton - Ecuador. This model constitutes a paradigm of internal and external organization at institutional level that allows to channel in an effective and timely manner, the guidelines to be applied within the educational field from different areas; a new restructuring is proposed that guarantees and ensures the fulfillment of the right to education, a path that will lead to quality and warmth to strengthen educational services, taking into account local and cultural realities, having an impact on the basic education schools. The research is divided into two types: descriptive, since a questionnaire of 10 questions was applied to 124 teachers belonging to the schools: Carlos Cueva Tamariz, Luís Roberto Chacón, Simón Bolívar, San José de Calasanz, Ezequiel Cárdenas and Santa Rosa de Lima from the city of Cañar, Ecuador. Documentary for the support of research through electronic and bibliographic means. Among some conclusions that can be mentioned, we have that the new model of educational management implemented in Ecuador in 2010, has an important incidence in the basic education schools of the city of Cañar, Cantón Cañar - Ecuador, since its actors in its Most of them know their guidelines and ratify the fulfillment of their objectives, especially in what has to do to strengthen the quality of education, by updating and strengthening the curricula of the General Basic Education and the Baccalaureate and the construction of the curriculum of Initial Education. And among the recommendations, share responsibilities and leadership to comply with the new model of educational management and bring them closer to citizenship, addressing local and cultural realities to adequately assume the assigned roles.
\end{abstract}

Key words: Decentralization, Education, Management, Organization. 


\section{Introducción}

Los sistemas educativos no cambian a la misma velocidad que se producen las modificaciones en los otros sistemas sociales, económicos, políticos, culturales, tecnológicos. Muchas veces estas estructuras sociales son reacias a transformarse, mediatizados por marcos organizativos y culturales, que privilegian la reproducción a la creación y la innovación. Esta misma situación, se reproducen a nivel de la escuela, sus docentes, el currículo. (Barros, 2012, p.29)

$\mathrm{Y}$ es lógico que esto suceda, hay que tener mucho cuidado, ya que se está hablando de renovar un sistema formador de ciudadanos que van a ser útiles para la sociedad y el desarrollo de la nación.

En ese sentido, debe hacerse un profundo análisis de la idiosincrasia de los países, y ha donde y con qué estrategias se quiere lograr la máxima eficiencia educativa, tomando en consideración que:

En el Ecuador, salvo pocos campos, experiencias y proyectos que arrojaron un saldo positivo, desde mediados de los ochenta y a lo largo de los noventa del siglo pasado, el saldo general fue negativo. Es así, que a finales de siglo el sistema educativo colapsó "frente a las narices" de los gobiernos de turno, y de la sociedad civil, a quienes se los llego a considerar responsables del fracaso del área educativa, por permitir la disminución sensible de las inversiones en educación, deteriorando la calidad, su infraestructura, la producción de material educativo, la profesión docente, la capacitación y formación profesional, la gestión y administración central y provincial, etc.(Paladines, 2015, p.13 y 14)

Para la ejecución de procesos educativos eficaces, también se requiere un esfuerzo añadido por parte de las escuelas para desarrollar intervenciones que tengan en cuenta las necesidades y realidades de todos los estudiantes. Para ello, una de las premisas fundamentales es que exista una adecuada comunicación entre los actores del sistema educativo y coherencia en un proyecto común. "Este clima de relación requiere un esfuerzo añadido por parte de las escuelas para desarrollar intervenciones que tengan en cuenta las necesidades y realidades de todos los estudiantes, y que desarrollen procesos educativos eficaces" (Alvarez, Aguirre, y Vaca, 2010, p.321).

De igual manera, es importante poner a deferencia el sentimiento de los padres al desear que a sus hijos se les proporcione una educación en muchos aspectos muy similares a las que ellos recibieron, y en el caso de padres que no tuvieron una buena formación educacional por diferentes razones, la idea es que sus hijos tengan acceso a una educación que les proporciones herramientas importantes para su desarrollo como ser humano. Siendo necesaria la participación e integración de alumnos, legisladores, investigadores y estudiosos de la educación, autoridades educativas locales, organizaciones de la sociedad civil, y de toda persona comprometida con las escuelas públicas y con el futuro de nuestro país.

$\mathrm{Si}$ bien, en las últimas décadas, en el Ecuador se han evidenciado cambios importantes en su estructura social, los sectores urbanos y rurales se han modificado, los sectores campesinos e indígenas se han promocionado y requieren servicios básicos. Este crecimiento no ha tenido las acciones correspondientes que contrarresten los problemas sociales que genera el desarrollo y conlleven a la consolidación del avance de la población, la crisis estructural resultado de la persistencia de un modelo educativo concentrador y excluyente han marginado de la vida social, económica y cívica a grandes grupos de población de zonas deprimidas, que, por carecer de una organización sólida, soportan los problemas de la injusticia social.

Por las aseveraciones expuestas, el estudio tiene por objetivo evaluar el nuevo modelo de gestión educativa implementado en el Ecuador y su impacto en las escuelas de educación básica del cantón Cañar - Ecuador. Considerando que modelos educativos anteriores ha generado depresión, dependencia y analfabetismo y sus consecuencias se manifiestan en el aumento de las tasas de desempleo y subempleo; bajos ingresos de la población; disminución del poder adquisitivo de la moneda y deterioro de la calidad de vida.

Aunque el objetivo general de los proyectos fue mejorar la calidad de la educación, debido al mal diseño o al bajo nivel de planificación es virtualmente imposible determinar si los alumnos en las escuelas seleccionadas aprendieron más que sus pares en otras escuelas. Sin embargo, los datos nacionales evidenciaron un significativo deterioro de las destrezas escolares entre 1996 y 2000, que es precisamente el período en el que se esperaba que las inversiones en el mejoramiento de la calidad hubiesen dado sus frutos.(FLACSO, 2008, p.69)

El deterioro de los Proyectos Educacionales ha sido alarmante. A continuación citamos algunos de los muchos problemas que se encuentran inmersos:

- Desigualdad en el acceso a la educación para los niveles pre-primario y medio se demuestran en las zonas urbana y rural.

- $\quad$ Escasez de oportunidades educativas para la población minusválida acorde con sus condiciones físicas y psicológicas; el analfabetismo adulto subsiste en cifras absolutas.

- Carencia de investigaciones para la generación de proyectos que permitan el mejoramiento cualitativo y cuantitativo de la educación.

- Inadecuación de los planes y programas de estudio a las posibilidades e intereses de los alumnos y a los avances de la ciencia y la tecnología.

- Deficiencias en la formación y capacitación de los docentes para la aplicación de concepciones y técnicas 
pedagógicas, en la actualización de conocimientos de iniciación científica y tecnológica.

- Malas condiciones de funcionamiento de los planteles por el elevado número de aulas en mal estado y los altos promedios alumno-aula por capacidad instalada insuficiente.

- Falta de un adecuado funcionamiento en la administración y coordinación del sistema educativo, en los niveles central y provincial.

- Los profesores que ejercen el magisterio al margen del mejoramiento profesional, generalmente no tienen la preparación adecuada para una práctica docente innovadora.

- La docencia en las condiciones poco favorables del medio rural, no permite incorporar positivamente, dentro del proceso de enseñanza aprendizaje, las experiencias y condicionamientos del contexto cultural y ambiental dentro del cual se desempeñan.

- El establecimiento de la jornada única ha ocasionado el detrimento de la calidad de enseñanza, por cuanto las tareas propiamente docentes se ven afectadas por la escasez de tiempo dedicado por el profesor (Organizacion de Estados Iberoamericanos, 1994).

Entre otros aspectos críticos (Flores, 2013) en su estudio indica que:

Los resultados de las pruebas censales SER Ecuador 2008 no son muy alentadoras. En el área de Matemáticas, el $81,96 \%$ de los estudiantes del tercer año de bachillerato tiene calificaciones entre regular e insuficiente, $80,43 \%$ los estudiantes del décimo año de Educación Básica, 55,48 \% los de séptimo y el $68,43 \%$ los de cuarto año. En lo referente a Lengua y Comunicación, el 50,37 \% de los estudiantes del tercer año de bachillerato tiene calificaciones entre regular e insuficiente, 53,31\% los estudiantes del décimo año de Educación Básica, 53,97\% los de séptimo y el 67,56\% los de cuarto año. Con respecto a Ciencias Naturales, el $82,98 \%$ de los estudiantes del décimo año de Educación Básica tiene calificaciones entre regular e insuficiente y el 49,58\% los de séptimo año. Finalmente en Ciencias Sociales, el 85,61\% de los estudiantes del décimo año de Educación Básica tiene calificaciones entre regular e insuficiente y el 74,5\% los de séptimo año. (pág. 10)

Surge entonces la necesidad de diseñar, implementar y evaluar un modelo educativo que supere todas debilidades anteriormente expuestas. En este contexto Ortiz (2010), el Dr. Carlos Ortega manifiesta que:

- Un modelo educativo implica la política educativa, la filosofía de la educación y la concepción teórica sobre educación con lo que se pretende lograr unidad de los códigos culturales y se concreta en la comunidad (participantes del hecho educativo).

- Desde el punto pedagógico un modelo implica el contenido de la enseñanza, el desarrollo del alumno y las características de la práctica docente para lograr aprendizajes y este se concreta en el aula (Ortega, 2014).

Estas posiciones constituyen referencias para la fundamentación de las redes educativas que garanticen una educación de calidad, humanista y socializadora para quienes cursan estudios y deben participar en el enriquecimiento de generaciones que garanticen un crecimiento cultural social y espiritual de la nación (Baquerizo, 2014, p.4).

En definitiva la educación es la base para la convivencia pacífica y respetuosa, para alcanzar una sociedad más justa y próspera. Todos los sectores sociales coinciden en señalar que es uno de los pilares del desarrollo, por tanto existe una relación directa entre la calidad del sistema educativo y las oportunidades de crecimiento de un país en su conjunto.

Se aspira el logro de una educación de calidad, con equidad e inclusión y que cumpla con los dos grandes propósitos de la educación básica, que son aprender a aprender y aprender a convivir, es necesario seguir en el proceso de revisión del modelo educativo, a fin de desechar los esquemas obsoletos y definir lo que es preciso mantener y potenciar en las escuelas de educación básica del Ecuador y precisamente en las escuelas de la ciudad de Cañar, Cantón Cañar, Ecuador.

\section{Reglamentación Ecuatoriana en el área educativa}

Los reglamentos para acrecentar la calidad de la educación en el Ecuador en los últimos años han experimentado cambios en miras a su desarrollo. Así el modelo educativo ecuatoriano ha pasado por 17 reformas, posiblemente el dato no sea exacto, porque en términos estrictos reforma es un cambio significativo que abarca varios niveles, que tiene un plazo por lo menos mediano y que debería integrar como mínimo, objetivos, procesos, cobertura nacional, mecanismos de evaluación y estudio de impacto, una reforma, supone una propuesta que involucre áreas como el financiamiento, formación inicial y permanente de los docente, contenidos, metodologías, material educativo, marco legal, sistema salarial, textos escolares, administración, etc. Es decir el estado ecuatoriano requiere de políticas educativas para el mediano y largo plazo.

Como sostiene la (Mesa de Educacion, 2002, p.2) en su enunciado "En el año 2002, la educación es colocada nuevamente en el escenario nacional y en la agenda electoral, fundamentalmente en torno al Contrato Social por la Educación, se plantea asegurar diez años de educación básica de calidad para todos los ecuatorianos". Sin embargo la inestabilidad política y social reinante en el país, crea un escenario inapropiado para que se desarrolle el proceso educativo.

A continuación, se revisa la reglamentación Ecuatoriana y escenarios en lo referente a la educación; el 26 de Noviembre de 2006, por mandato ciudadano, se convierte en política de estado, El Plan Decenal de Educación 20062015, aprobado por más del $66 \%$ de los votantes, sin 
embargo el voto blanco tubo una significativa incidencia, pues la falta de financiamiento no permitió realizar una difusión adecuada del sentido de la propuesta.

El Plan Decenal de Educación de acuerdo a sus lineamientos, considera que, el pilar fundamental del ser humano es la educación y "garantiza la calidad de la educación nacional con equidad, visión intercultural e inclusiva, desde un enfoque de los derechos y deberes para fortalecer la formación ciudadana y la unidad en la diversidad de la sociedad ecuatoriana" como determina el objetivo general del Plan Decenal de Educación 2006-2015 (Ministerio de Educación, 2016; Rodríguez, 2017).

En resumen el mandato ciudadano obliga a institucionalizar El Plan Decenal de Educación, con sus programas, objetivos y metas construidos en el marco de una política de estado. Al respecto el Ministro de Educación, en ese entonces (año 2008) Raúl Vallejo Corral manifiesta que "ciertamente las políticas educativas por sí solas no modifican las inequidades sociales que permanecen en nuestra sociedad pero, no es menos cierto, una educación pública de calidad y calidez para el conjunto de la población sí contribuye a generar la esperanza de una vida mejor en las personas. Y es que de eso se trata: como país necesitamos construir un sistema educativo que, en síntesis, sea capaz de ofrecer una educación de la más alta condición académica en América Latina y el mundo y que forme una ciudadanía socialmente responsable, plena de valores éticos y estéticos" (Consejo Nacional de Educacion, 2007, p.4). Y, para garantizar y asegurar el cumplimiento del derecho a la educación, el Nuevo Modelo de Gestión Educativa (NMGE) es un proyecto que inició su gestión en enero de 2010, y plantea la reestructuración del Ministerio de Educación. Busca influir de manera directa sobre el acceso universal y con equidad a una educación de calidad y calidez, lo que implica ejecutar procesos de desconcentración desde la planta central hacia las zonas, distritos y circuitos, y así fortalecer los servicios educativos y aproximarlos hacia la ciudadanía, atendiendo las realidades locales y culturales, constituyéndose en una nueva práctica de realización del servicio público (mejor distribución de personal capacitado e idóneo); así como la racionalización de recursos, distribución de competencias y responsabilidades (Ministerio de Educación, 2018a).

Además, la Subsecretaría de Apoyo, Seguimiento y Regulación de la Educación (ASE) determina el nuevo esquema organizacional de la conducción de la educación, establece trabajar con los siguientes cinco niveles de desconcentración:

- Nivel central, con las facultades de rectoría, regulación y planificación;

- Nivel zonal, con las facultades de regulación, planificación, coordinación y control de los servicios educativos de su zona;

- Nivel distrital, con las facultades de planificación, coordinación, control y gestión de los proyectos y servicios educativos de su distrito, oferta de información a los diferentes niveles y modalidades educativas del territorio;

- Nivel de circuito, con facultades de planificación y gestión, brinda acompañamiento pedagógico a las instituciones educativas y docentes;

- Instituciones educativas, con facultades de planificación y gestión de su institución” (ASE, 2013, p.10, 11).

La descentralización del sistema educativo mediante la creación de redes escolares fue vista como un camino que conduciría de la cobertura a la calidad, y representó el mayor esfuerzo por mejorar la educación pública en el país durante los últimos quince años.

En ese período es posible señalar una lista de cambios: nuevas escuelas, nuevas aulas, libros y material didáctico, reuniones de capacitación, un nuevo currículo para la educación básica y principalmente es importante destacar a un Ministerio de Educación que trabaja para la ciudadanía, incrementando procesos desconcentrados, ágiles, transparentes y automatizados, eliminando procesos burocráticos, centralizados, poco eficientes y con poco acceso ciudadano.

Un importante logro alcanzado fue "la aprobación y expedición del nuevo Estatuto Orgánico de Gestión Organizacional por Procesos del MINEDUC, mediante acuerdo ministerial 020-12 del 25 de enero del 2012. Adicionalmente, se cuenta con el manual de clasificación, descripción y valoraciones de puestos institucionales, en esa medida la optimización del Talento Humano, es decir, los ingresos por méritos, movimientos, traspasos, traslado y desvinculaciones del personal, serán posibles, con la aplicación de la evaluación por reestructura para servidores públicos con nombramiento de planta central y direcciones provinciales hispanas y bilingües, bajo régimen de la LOSEP. (Ministerio de Educación, 2016)

Por otro lado la Ley Orgánica de Educación Intercultural (LOEI) determina que el Ministerio se integrará preferentemente con los actuales funcionarios y empleados, previo un proceso de selección que estará a cargo de la Dirección de Recursos Humanos" (Illescas, 2013, p.20).

Por otro lado, la consolidación de un nuevo Modelo Nacional de Apoyo y Seguimiento a la Gestión Educativa (MNASGE) constituye una de las metas del Plan Decenal y forma parte de la política seis, que se propone el mejoramiento de la calidad y equidad de la educación y la implementación de un sistema nacional de evaluación. La segunda línea de acción de esta política establece el "Desarrollo de modelos pedagógicos que evolucionen y se adapten a las necesidades socioculturales y de desarrollo nacional" (ASE, 2013, p.3).

En este ámbito el estado, a través de su Gobierno, tiene la obligación de garantizar la calidad del servicio educativo que reciben como un derecho constitucional los niños, niñas y jóvenes ecuatorianos. Para lograrlo, el Ministerio de Educación del Ecuador, a través de la Subsecretaría de Apoyo, Seguimiento y Regulación Educativa (SASRE) requiere de un sistema que permita asegurar que las instituciones educativas asuman la mejora como un proceso necesario, 
cíclico, permanente, sistemático e integrado a la gestión que despliegan (ASE, 2013, p.3).

En consecuencia, la Subsecretaría de Apoyo, Seguimiento, y Regulación con el apoyo de la VVOB diseñó el Modelo Nacional de Apoyo y Seguimiento a la Gestión Educativa (MNASGE) y ha iniciado su implementación. La aplicación de este modelo es posible a través de las acciones orientadoras de dos instancias. Una, denominada de asesoría, y otra, denominada de auditoría educativa (ASE, 2013, p.3).

En conclusión el Nuevo Modelo de Gestión favorece la consecución de servicios educativos en zonas centrales y cercanas a la colectividad, brindando mayor eficiencia, rapidez y cobertura. En las 9 zonas se implementaron alrededor de 140 direcciones distritales a nivel nacional para atención a la población, y 1200 circuitos educativos, aproximadamente, con los cuales se garantiza, una oferta completa de servicios educativos (Quishpe, 2017, p.15). Contempla:

- Administración escolar que implica, alimentación escolar, textos, uniformes, infraestructura, equipamiento, mobiliario (Subsecretaría de Administración Escolar, 2018).

- Concursos de méritos y oposición, cuyo objetivo es la regularizar la relación laboral de los docentes por contrato, participan aspirantes para ingresar a la carrera educativa pública y los docentes a los que les corresponda hacerlo por solicitud de traslado del lugar de trabajo (Ministerio de Educacion, 2018).

- Educación para la democracia y el buen vivir, propone políticas para la implementación efectiva de prácticas de democracia y buen vivir.

- Estándares de calidad educativa, sirven para orientar, apoyar y monitorear la gestión de los actores del sistema educativo hacia su mejoramiento continuo. Entre estos tenemos: los de gestión escolar; que constituyen prácticas institucionales que contribuyen a la formación deseada de los estudiantes, de desempeño profesional; que son descripciones de lo que debe hacer un profesional educativo competente, estándares de aprendizaje; que se refieren a los logros de aprendizaje que los estudiantes deben alcanzar a lo largo de la trayectoria escolar y los de infraestructura escolar que establecen requisitos esenciales, orientados a determinar las particularidades que los espacios y ambientes escolares deben poseer para contribuir al alcance de resultados óptimos en la formación de los estudiantes y en la efectividad de la labor docente.

- Fortalecimiento del Inglés, cuyo objetivo principal el que los estudiantes ecuatorianos alcancen un nivel funcional de uso de la lengua inglesa (Ministerio de Educación, 2018b).

- Sistema Nacional de Evaluación que implica el monitoreo de la calidad de la educación que brinda el sistema educativo ecuatoriano y la definición de políticas que permitan mejorar los procesos de enseñanza y aprendizaje (Sistema Nacional de Evaluación y Rendición Social de Cuentas, 2008, p.7).

- Formación docente, que implica cursos y talleres de formación.

- Unidades del milenio cuyo objetivo fundamental es brindar una educación de calidad y calidez, mejorar las condiciones de escolaridad, el acceso y la cobertura de la educación en sus zonas de influencia, y desarrollar un modelo educativo que responda a las necesidades locales y nacionales (Soto, Sánchez, Martillo, y Sarmiento, 2015, p.22).

\section{Metodología}

El presente estudio alcanzo un tipo de investigación descriptiva y documental. Descriptiva porque se limitó a describir el fenómeno estudiado, esto es, el nuevo modelo de gestión educativa y su impacto en las escuelas de educación básica del cantón Cañar - Ecuador. A su vez, se aplicó un cuestionario de 10 preguntas cerradas sobre el objeto de la investigación. El universo de esta investigación estuvo integrado por 124 docentes pertenecientes a las escuelas: Carlos Cueva Tamariz, Luís Roberto Chacón, Simón Bolívar, San José de Calasanz, Ezequiel Cárdenas y Santa Rosa de Lima de la ciudad de Cañar, cantón Cañar Ecuador. Documental ya que se ha recurrido a información secundaria proveniente de medios bibliográficos, electrónicos y revistas de investigación, donde se han recopilado conceptos y opiniones importantes de otros investigadores para fundamentar la investigación.

\section{Resultados}

Como resultado de la aplicación del cuestionario a los docentes de las escuelas de educación básica del Cantón Cañar se verificó que:

1) La información socioeconómica obtenida de los encuestados, demostró que de los 124 docentes encuestados, 92 de ellos $(74 \%)$ corresponden al género femenino y los 32 restantes $(26 \%)$ pertenecen al género masculino.

2) En cuanto al nivel de instrucción, 9 docentes (7\%) solamente tienen estudios de cuarto nivel, 82 (66\%) poseen títulos de pregrado y $33(27 \%)$ son bachilleres.

En lo que se refiere a las preguntas de cuestionario se determina que:

1) El $86 \%$ de los docentes encuestados manifiestan conocer el nuevo modelo de gestión educativa, dentro de este indicador el $71 \%$ están de acuerdo con el mismo.

2) Los docentes opinan que están de acuerdo con el nuevo modelo de gestión educativa, porque: fortalece la calidad educativa, promueve la participación ciudadana, proporciona procesos educativos basados en equidad, inclusión y permanencia, además proporciona un bajo nivel de descentralización y finalmente indican que genera alta desconcentración de la gestión educativa. 
3) El $81 \%$ de los educadores conocen el marco de acción del Programa Nacional de Educación para la Democracia y el Buen Vivir.

Es así, que el $90 \%$ de los maestros, opinan que los estándares de gestión educativa contribuyen a que los estudiantes logren los resultados de aprendizaje esperados. En este ámbito apenas el $68 \%$ de los docentes manifiestan que los estándares de gestión educativa contribuyen a que las instituciones educativas se aproximen a su funcionamiento óptimo y que sus actores se desarrollen.

En cuanto a los estándares de desempeño profesional, de acuerdo a la distribución de los datos, el $87 \%$ de los encuestados indican conocer cuáles son sus características.

Por otro lado el $86 \%$ de los docentes, identifican las áreas básicas que comprenden los estándares de aprendizaje.

El $81 \%$ de los encuestados conocen, los componentes que forman parte del Sistema Nacional de Evaluación.

En lo referente al sistema de Desarrollo Profesional Educativo (SIPROFE), el $78 \%$ de los educadores están de acuerdo con sus lineamientos, y manifiestan que contribuye a su formación continua y busca afianzar la calidad del sistema educativo.

\section{Conclusiones}

El Nuevo Modelo de Gestión Educativa implementado en el Ecuador en el año 2010, tiene una incidencia importante en las escuelas de educación básica de la ciudad de Cañar, Cantón Cañar - Ecuador, pues sus actores en su gran mayoría conocen sus lineamientos y ratifican el cumplimiento de sus objetivos, especialmente en lo que tiene que ver al fortalecimiento de la calidad de la educación, mediante la actualización y fortalecimiento de los currículos de la Educación General Básica y del Bachillerato y la construcción del currículo de Educación Inicial. Como complemento de esta estrategia, y para facilitar la implementación del currículo, se han elaborado nuevos textos escolares y guías para docentes. Dicho programa se sustenta en los principios de la Pedagogía Crítica, que coloca al estudiante como el actor principal del aprendizaje.

La ejecución de procesos de desconcentración desde la Planta Central hacia las zonas, distritos y circuitos ha fortalecido los servicios educativos, atendiendo la realidad local y cultural de nuestra sociedad.

Los procesos basados en equidad, inclusión y permanencia se ponen de manifiesto en las escuelas de educación básica del Cantón Cañar, eliminando la disparidad educativa particularmente en grupos vulnerables a la discriminación y la exclusión, en este sentido se han incluido niñas, niño/as pobres, niño/as trabajadores y de la calle, población rural, minorías étnicas, población con discapacidad y otros grupos.

En cuanto al Programa Nacional de Educación para la Democracia y el Buen Vivir, la implementación de los Departamentos de Consejería Estudiantil previene la deser- ción y ejecuta acciones de prevención frente a situaciones de vulneración de derechos de niños, niñas y adolescentes.

En relación a los estándares de gestión educativa en las escuelas de educación básica del Cantón Cañar, si bien contribuye a que los estudiantes logren los resultados de aprendizaje esperados. Se debe mejorar los procedimientos para que las instituciones educativas se aproximen a su funcionamiento óptimo y sus actores se desarrollen.

Los estándares de desempeño profesional, permite reflexionar, evaluar y autoevaluar para diseñar y ejecutar estrategias de mejoramiento, por otro lado los estándares de aprendizaje están plenamente identificados por los docentes en cuatro áreas básicas: Lengua y Literatura, Matemática, Ciencias Sociales y Ciencias Naturales.

Los procesos de enseñanza y aprendizaje que se ejecutan en cada una de las instituciones educativas inmersas en este estudio, son monitoreados por el Sistema Nacional de Evaluación con la finalidad de evaluar la calidad de la educación que brindan.

Finalmente un porcentaje significativo $(78 \%)$ de los docentes de las escuelas de educación básica de la ciudad de Cañar - Ecuador mira al programa de Desarrollo Profesional Educativo (SIPROFE) como la guía que permite establecer nuevas rutas, con base en la evaluación del desempeño de cada uno de ellos.

\section{Recomendaciones}

Se recomienda a la Coordinación Zonal 6 (Azuay, Cañar, Morona Santiago):

Realizar capacitaciones oportunas a los miembros de la comunidad educativa para socializar, el nuevo modelo de gestión educativa y sus programas, especialmente en lo que se refiere a: Programa Nacional de Educación para la Democracia y el Buen Vivir, estándares de desempeño profesional, estándares de aprendizaje y componentes del Sistema Nacional de Evaluación, de manera que sea conocido por todos y cada uno de sus actores.

Implementar el modelo de gestión educativa, acorde a las realidades e intereses del entorno socio-cultural del Cantón Cañar.

Compartir responsabilidades y liderazgos para dar cumplimiento al nuevo modelo de gestión educativa y aproximarlos hacia la ciudadanía, atendiendo las realidades locales y culturales para asumir adecuadamente los roles asignados.

A la Universidad Católica de Cuenca Extensión Cañar:

Que a través de sus programas de vinculación con la colectividad, se designe profesionales y estudiantes, para organizar talleres, foros, reuniones, etc. sobre el nuevo modelo de gestión educativa y sus implicaciones en las escuelas de educación básica del Cantón Cañar.

Se recomienda a los actores de la comunidad educativa:

Participar activamente en el logro de una educación de calidad, cumplir con los estándares de calidad educativa, 
que sirven para orientar, apoyar y monitorear su gestión hacia el mejoramiento continuo.

\section{Referencias Bibliográficas}

Alvarez, B., Aguirre, M., y Vaca, S. (2010). Interacción Familia-Escuela. Análisis de contenido sobre el discurso de docentes y familias (Investigación desarrollada en Centros Educativos de Ecuador). Revista española de orientación y psicopedagogía, 320-334.

ASE. (2013). Modelo Nacional de Apoyo y Sseguimiento (Inf. Téc.).

Baquerizo, R. (2014). La calidad de la gestion educativa. Revista Científica ECOCIENCIA, 1-21.

Barros, J. M. (2012). Indicadores de calidad educativa en centros escolares del Distrito Metropolitano de Quito y propuesta de un modelo de calidad pertinente para el Ecuador (Inf. Téc.).

Consejo Nacional de Educacion. (2007). Plan Decenal de Educacion del Ecuador 2006-2015. Año 2 de su Ejecucion (Inf. Téc.).

Contreras, A., y Muñoz, V. (2016). Estado actual de los planes de mejora en los Centros de Desarrollo Infantil Privados (Inf. Téc.).

FLACSO. (2008). Desafíos de la educación en el Ecuador: calidad y equidad. Quito: Autor.

Flores, J. (2013). Incidencia de la Planificación Estratégica en la gestión de la calidad de los ámbitos de la gerencia educativa (Inf. Téc.).

Illescas, S. (2013). Evaluación del modelo de gestión del proyecto emblemático nacional"Unidades educativas del Milenio y su impacto de la política pública educativa. Caso Ecuador-Cotopaxi del 2008 al 2012 (Inf. Téc.). Quito.

Mesa de Educacion. (2002). PROPUESTA DE LA MESA DE EDUCACION. Descargado de http://www.filo.uba.ar/contenidos/ carreras/educa/catedras/educacion1/ sitio/Ecuador.htm

Ministerio de Educación. (2016). Ministerio de Educacion. Descargado de http://educacion.gob.ec/ reestructuracion-institucional/

Ministerio de Educación. (2018a). Ministerio de Educacion. Descargado de https:// educacion.gob.ec/que-es-el-nuevo -modelo-de-gestion-educativa/

Ministerio de Educación. (2018b). Ministerio de Educacion. Descargado de https:// educacion.gob.ec/fortalecimiento -del-ingles-prin/

Ministerio de Educacion. (2018). Ministerio de Educacion. Descargado de https: / / educacion.gob.ec/ informacion-concursos-de-meritos-y -oposicion/

Organizacion de Estados Iberoamericanos. (1994). Sistemas Educativos Nacionales - Ecuador (Inf. Téc.).

Paladines, C. (2015). Perspectivas de cambio en la Educación Básica y en el Bachillerato: Ecuador: 20072013. Praxis educativa, 13-31.

Quishpe, L. (2017). Los procesos de reclutamiento y selección del personal administrativo y de servicio en el distrito de educación 06d04 Colta-Guamote, y su influencia en el desarrollo institucional periodo 2014 - 2015 (Inf. Téc.).

Rodríguez, M. (2017). Unidades educativas del Milenio, educación intercultural bilingüe y (des) igualdad en el acceso a la educación en Ecuador: Un análisis desde la investigación etnográfica. Runa, 41-55.

Sistema Nacional de Evaluación y Rendición Social de Cuentas. (2008). (Inf. Téc.). Quito.

Soto, S., Sánchez, T., Martillo, E., y Sarmiento, C. (2015). Calidad educativa en Ecuador. Calidad Educativa.

Subsecretaría de Administración Escolar. (2018). Descargado de https: / / educacion.gob.ec/ wp-content/uploads/downloads / 2014/03/9.2-SUBSECRETARIA-DE -ADMINISTRACION-ESCOLAR.pdf

Recibido: 9 de enero de 2018

Aceptado: 1 de agosto de 2018 
